\title{
Methylene tetrahydrofolate reductase (MTHFR) gene rs1801133 $C>T$ polymorphisms and response to 5-FU based chemotherapy in patients with colorectal cancer: a meta-analysis
}

https://doi.org/10.1515/pteridines-2019-0015

received December 20, 2018; accepted April 25, 2019.

\begin{abstract}
Background: Methylene tetrahydrofolate reductase (MTHFR) catalyzes the conversion of 5,10-methylenetetrahydrofolate to 5-methyltetrahydrofolate, a cosubstrate for homocysteine remethylation to methionine. Single nucleotide polymorphisms (SNP) of MTHF rs1801133 C $>$ T can influence susceptibility to colorectal cancer. However, an association between MTHFR rs1801133 C>T polymorphisms and response to 5-Fluorouracil (5-FU) based chemotherapy in patients with colorectal cancer was not clear. Methods: Studies relevant to MTHFR rs1801133 C >T polymorphisms and response to 5-FU based chemotherapy in patients with colorectal cancer were systematic searched in the electronic databases of PubMed, Web of Science, Embase, and China National Knowledge Infrastructure (CNKI). The genotypes of CC, CT, and TT were extracted from each included publication. The genotypes CC, CT, and TT distribution in 5-FU based chemotherapy response and resistance groups were calculated and pooled through random or fixed effect model by the effect size of odds ratio (OR) and 95\% confidence interval $(95 \% \mathrm{CI})$. The publication bias was evaluated through Begg's funnel plot and Egger's line regression test. Results: After searching the electronic databases, 16 studies related to MTHFR gene rs1801133 C > T polymorphisms and a response to 5-FU based chemotherapy in patients with colorectal cancer were included in the present meta-analysis. The pooled data showed no statistical difference in tumor response
\end{abstract}

\footnotetext{
*Corresponding author: Yi Shen, Department of Colorectal Surgery, Shaoxing People“ Hospital (Shaoxing Hospital, Zhejiang University School Of Medicine), Shaoxing City Zhejiang Province 312000 PR China, E-mail: jfmjussxio@sohu.com

Huafeng Jiang, Department of Colorectal Surgery, Shaoxing People، Hospital (Shaoxing Hospital, Zhejiang University School Of Medicine), Shaoxing City Zhejiang Province 312000 PR China
}

rate between $\mathrm{CT}+\mathrm{TT}$ and $\mathrm{CC}$ groups in the dominant genetic model CT+CC vs CC (OR=1.21, 95\% CI: 0.93 1.59, $\mathrm{p}>0.05)$ and recessive model TT vs CT+CC (OR=1.37, 95\% CI: 0.91 2.06, $p>0.05)$. The grade 3-4 adverse reaction rate between CT+TT and CC groups also had no statistical difference in the dominant genetic model CT+CC vs CC (OR $=0.90,95 \%$ CI: 0.76 1.07, $\mathrm{p}>0.05)$ and recessive model TT vs CT+CC (OR=1.12, 95\% CI: 0.84 1.50, $\mathrm{p}>0.05)$. The Begg's funnel plot and Egger's line regression test demonstrated no publication bias. Conclusion: The response and adverse reaction of 5-FU based chemotherapy in colorectal patients were not different in terms of MTHFR rs1801133 C >T polymorphisms.

Keywords: MTHFR; polymorphism; chemotherapy; 5-FU; adverse reaction; meta-analysis.

\section{Introduction}

Methylene tetrahydrofolate reductase (MTHFR) is a key enzyme in folate metabolism and methionine metabolism. The rs1801133 C >T polymorphism of the MTHFR gene lead to a decrease in enzyme activity, inhibit the folic acid metabolism cycle, affect the synthesis of pyrimidine or purine, and lead to the damage of DNA synthesis or repair, as well as the defect or canceration of cell growth [1]. In recent years, with the development of pharmacogenomics, researchers began to explore the relationship between MTHFR gene SNPs and the sensitivity to chemotherapy regimens. 5-FU based chemotherapy regimen is extensive and used long-term clinically for esophageal-gastric cancer [2] and colorectal carcinoma [3, 4]. However, most of the studies relevant MTHFR rs1801133 C $>\mathrm{T}$ polymorphisms and response to 5 -FU based chemotherapy in patients with colorectal cancer were retrospective studies with small sample size. Therefore, we performed this meta-analysis 
by pooling the open published studies of the relationship between MTHFR rs1801133 C>T polymorphisms and 5-FU based chemotherapy response in order to provide a more reliable evidence for the rational use of 5-FU chemotherapy regimen in clinical practice.

\section{Material and methods}

\section{Publications searching and inclusion}

The electronic databases of PubMed, Web of Science, Embase, and CNKI were systematic searched to identify relevant studies related to MTHFR gene rs1801133 $\mathrm{C}>\mathrm{T}$ polymorphisms and a response to $5-\mathrm{FU}$ based chemotherapy in patients with colorectal cancer (Figure 1). The searching text words were methylene tetrahydrofolate reductase/MTHFR, colorectal cancer/colon cancer/rectal cancer/colorectal carcinoma/ colon carcinoma/ rectal carcinoma/ OR CRC, and chemotherapy. The publication screening procedure was last performed in October 10, 2018. The study inclusion criteria was (1) the patients were diagnosed of colorectal cancer by pathology, (2) the chemotherapy regimen was 5-FU based,(3) genotype of $\mathrm{CC}, \mathrm{CT}$, and TT can be extracted from original publications, and (4) tumor response rate and adverse reaction can be calculated from original studies of different genotype.

Ethical approval: The conducted research is not related to either human or animals use.

\section{Data extraction from original publication}

After reviewing the included full text paper, the general information and useful data was extracted by two reviewers independently and checked by a third reviewer as described according to the Cochrane Handbook for systematic reviews. The general information included the name of the authors, the manuscript publication year, region, chemotherapy regimen, and chemotherapy adverse reaction and response. The useful data of sample size, distribution of CC, CT, TT genotype in tumor response and resistance groups were carefully extracted from the original publications.

\section{Statistical methods}

STATA/SE 11.0 StataCorp LP, http://www.stata.com software were used for the data analysis. The odds of CC, CT and TT genotypes in chemotherapy sensitivity groups

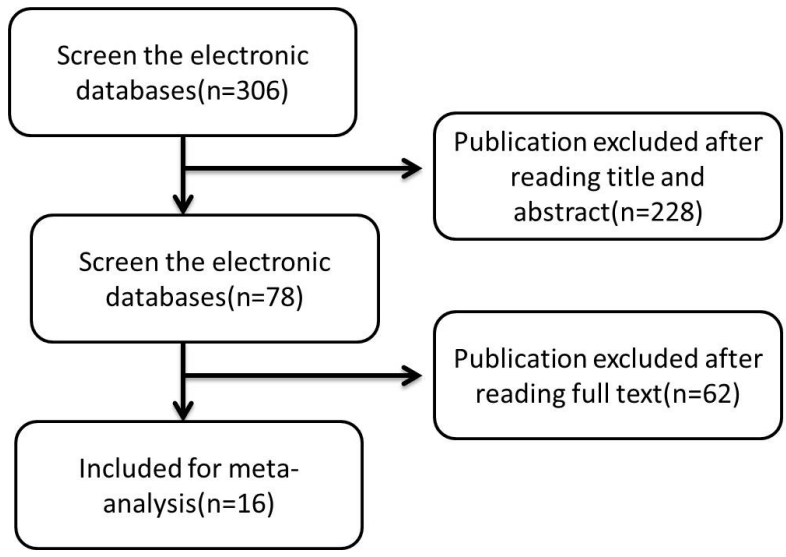

Figure 1: Literature screening process and results.

versus chemotherapy resistance groups, or grade 3-4 adverse reaction groups versus non-grade 3-4 adverse reaction groups were expressed by odds ratio (OR) and $95 \%$ confidence intervals (95\% CI). The statistical heterogeneity across the included 16 publications in each effect size was assessed by I ${ }^{2}$ test [5]. The OR was pooled by random or fixed effect models according to statistical heterogeneity. The publication bias was assessed by Begg's funnel plot and Egger's line regression tests [6].

\section{Results}

\section{General information of the included publications}

After searching the electronic databases of PubMed, Web of Science, Embase, and CNKI, 16 studies [7-22] related to MTHFR gene rs1801133 C $>\mathrm{T}$ polymorphisms and response to 5 -FU based chemotherapy in patients with colorectal cancer were included in the present meta-analysis. The general information of the included 16 publications is shown in Table 1.

\section{Tumor response rate in dominant mutation model $[(\mathrm{CT}+\mathrm{TT})$ vs $\mathrm{CC}]$}

For dominant genetic model [(CT+TT) vs CC], there was no significant statistical heterogeneity $\left(\mathrm{I}^{2}=42.2 \%, \mathrm{P}=0.07\right)$. Therefore, the data was pooled under a fixed effect model. The combined results demonstrated there was no statistical difference in tumor response rate between CT+TT and CC groups (OR=1.21, 95\% CI: 0.93 1.59, $\mathrm{p}>0.05$ ), Figure 2. 
Table 1: General characteristics of the included 16 studies.

\begin{tabular}{|c|c|c|c|c|c|c|c|c|}
\hline \multirow[t]{2}{*}{ First author } & \multirow{2}{*}{$\begin{array}{l}\text { Publication } \\
\text { (year) }\end{array}$} & \multirow[t]{2}{*}{ Region } & \multirow{2}{*}{$\begin{array}{l}\text { Sample } \\
\text { size }\end{array}$} & \multicolumn{3}{|c|}{ Genotype } & \multirow[t]{2}{*}{ Chemotherapy } & \multirow[t]{2}{*}{ Results } \\
\hline & & & & CC & CT & TT & & \\
\hline Cohen & 2003 & Canada & 64 & 17 & 42 & 5 & 5FU/Capecitabine & TR \\
\hline Etienne & 2004 & France & 97 & 52 & 27 & 18 & 5-FU/LV & TR \\
\hline Jakobsen & 2005 & Denmark & 79 & 32 & 38 & 9 & 5-FU/LV & TR \\
\hline Marcuello & 2006 & Spanish & 94 & 32 & 50 & 12 & FOLFOX/FOLFIRI & TR \\
\hline Capitain & 2007 & France & 67 & 28 & 31 & 8 & 5-FU/LV & TR \\
\hline Ruzzo & 2008 & Italia & 146 & 40 & 73 & 33 & FOLFIRI & TR \\
\hline Suh & 2008 & Korea & 50 & 16 & 28 & 6 & FOLFOX & TR \\
\hline Sharma & 2008 & Australia & 54 & 27 & 20 & 7 & FLFOX/FOLFIRI & AR \\
\hline Braun & 2009 & England & 1601 & 734 & 719 & 148 & FU/FLFOX/FOLFIRI & $A R$ \\
\hline Chua & 2009 & Australia & 116 & 43 & 54 & 19 & FOLFOX & $\mathrm{AE}$ \\
\hline Etienne-Grimaldi & 2010 & France & 116 & 44 & 58 & 14 & FOLFOX & TR \\
\hline Glimelius & 2011 & Sweden, Norway & 140 & 68 & 63 & 9 & FLIRI/FOLIRI & $A R$ \\
\hline van Huis-Tanja & 2013 & Netherlands & 264 & 106 & 131 & 27 & Capecitabine /XELIRI & $A R$ \\
\hline Kumamoto & 2013 & Japan & 62 & 26 & 30 & 6 & mFOLFOX6 & AR TR \\
\hline Shen & 2015 & China & 127 & 51 & 60 & 16 & FOLFOX & TR \\
\hline Dong & 2016 & China & 72 & 39 & 30 & 3 & FOLFOX6 & TR \\
\hline
\end{tabular}

TR: tumor response; AR: adverse reaction

Study

ID

Capitain (2007)

Dong (2016)

Etienne-Grimaldi (2010)

Kumamoto K (2013)

Marcuello (2006)

Suh (2008)

Shen (2015)

Etienne (2004)

Cohen (2003)

Jakobsen (2005)

Ruzzo (2008)

Overall (I-squared $=42.2 \%, p=0.068$ )

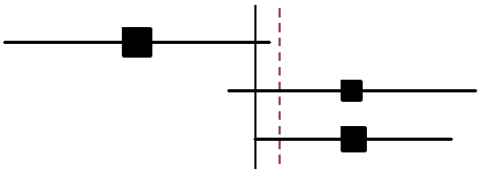

$0.40(0.14,1.12) 11.62$

$2.12(0.81,5.55) 5.85$

$2.14(1.00,4.60) 9.29$

$0.74(0.27,2.06) 8.80$

$0.78(0.33,1.83) 12.26$

$0.71(0.20,2.61) 5.78$

$2.16(1.02,4.58) 9.76$

$0.73(0.32,1.67) 13.42$

$3.05(0.84,11.07) 2.69$

$0.81(0.31,2.12) 9.49$

$1.59(0.74,3.42) 11.04$

$1.21(0.93,1.59) \quad 100.00$

\begin{tabular}{cc:cc} 
& & \\
\hline & & \\
0904 & 1 & 11.1
\end{tabular}

Figure 2: Forest plot of MTHFR gene rs1801133 C>T polymorphisms and chemosensitivity of colorectal cancer in dominant genetic model $[(\mathrm{CT}+\mathrm{TT})$ vs $\mathrm{CC}]$. 


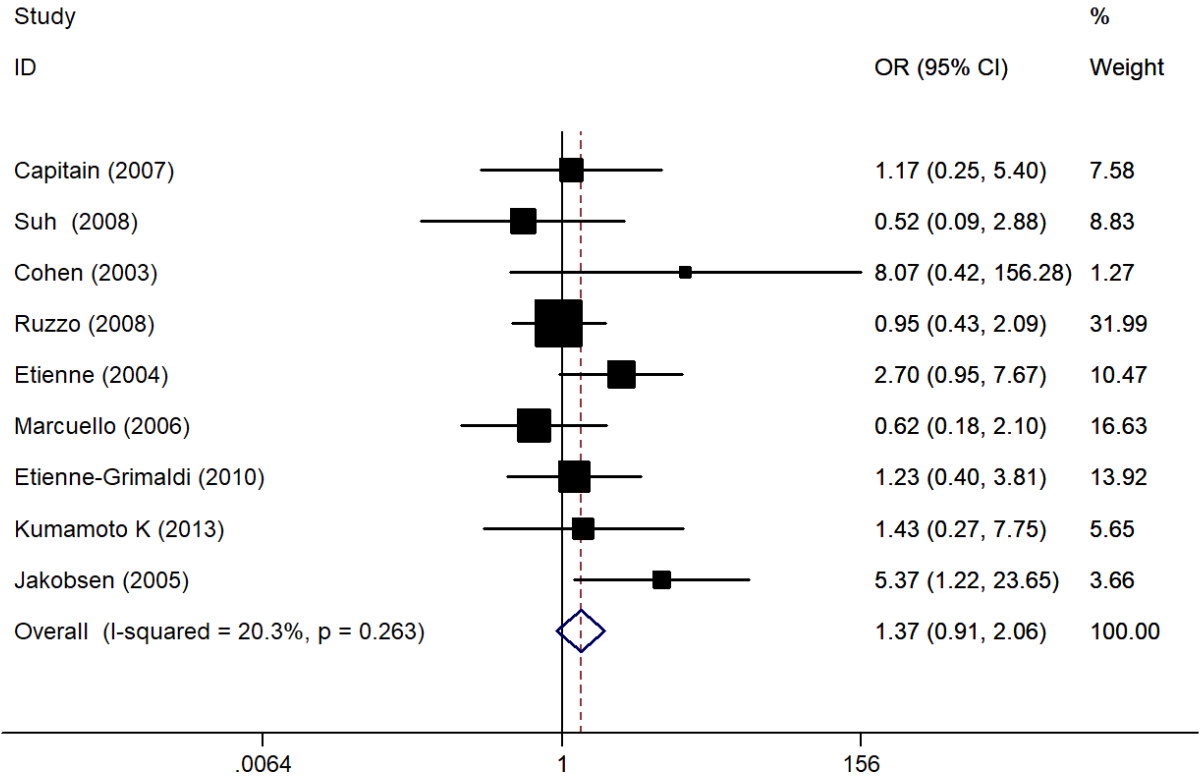

Figure 3: Forest plot of MTHFR gene rs1801133 C>T polymorphisms and chemosensitivity of colorectal cancer in recessive genetic model [(TT vs $(\mathrm{CT}+\mathrm{CC})]$.

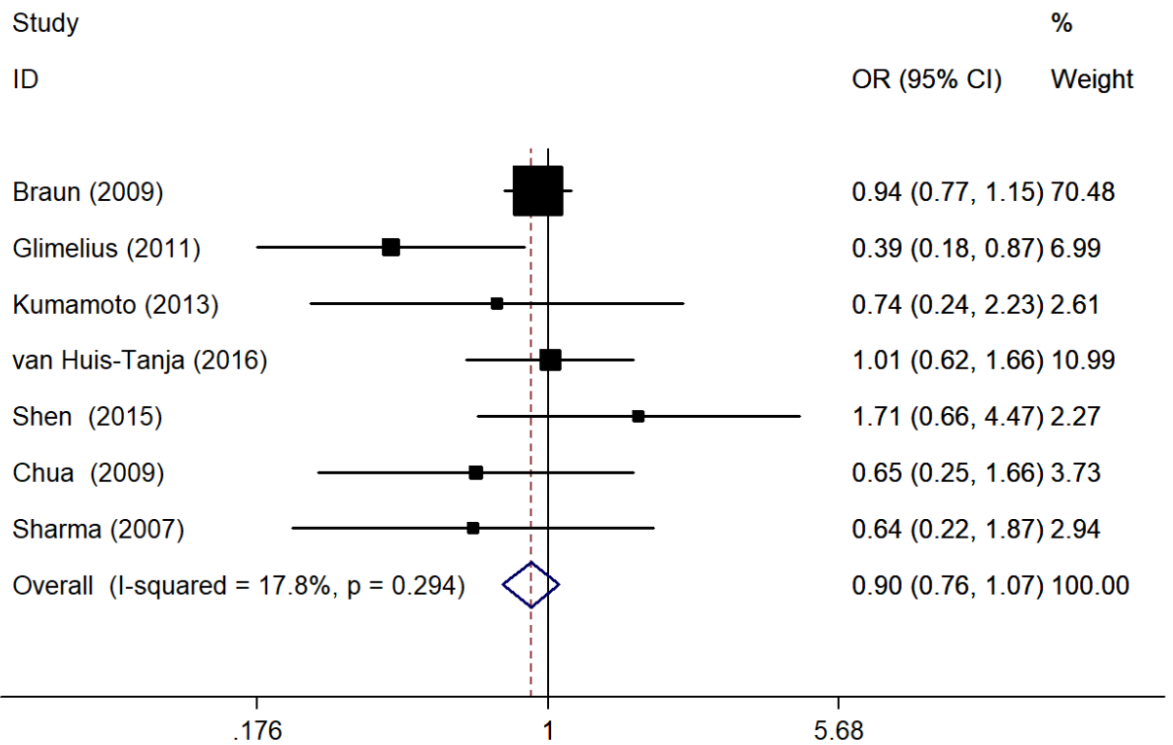

Figure 4: Forest plot of MTHFR gene rs1801133 C>T polymorphisms and grade 3-4 adverse reaction of colorectal cancer in recessive genetic model [dominant genetic model (CT+TT) vs CC].

\section{Tumor response rate in recessive mutation model [TT vs $(\mathrm{CT}+\mathrm{CC})]$}

For recessive genetic model [TT vs $(\mathrm{CT}+\mathrm{CC})]$, the pooled OR for tumor response rate between TT and CT+CC groups was (OR=1.37, 95\% CI: 0.91 2.06, p>0.05) in a fixed effect model without statistical difference, Figure 3.

\section{Grade 3-4 adverse reaction in dominant mutation model[(CT+TT) vs CC]}

For dominant genetic model [(CT+TT) vs CC], there was no significant statistical heterogeneity $\left(\mathrm{I}^{2}=17.8 \%, \mathrm{p}=0.29\right)$. The pooled data indicated no statistical difference of grade 3-4 adverse reaction in (CT+TT) and CC group with the (OR=0.90, 95\% CI: 0.76 1.07, p>0.05), Figure 4. 


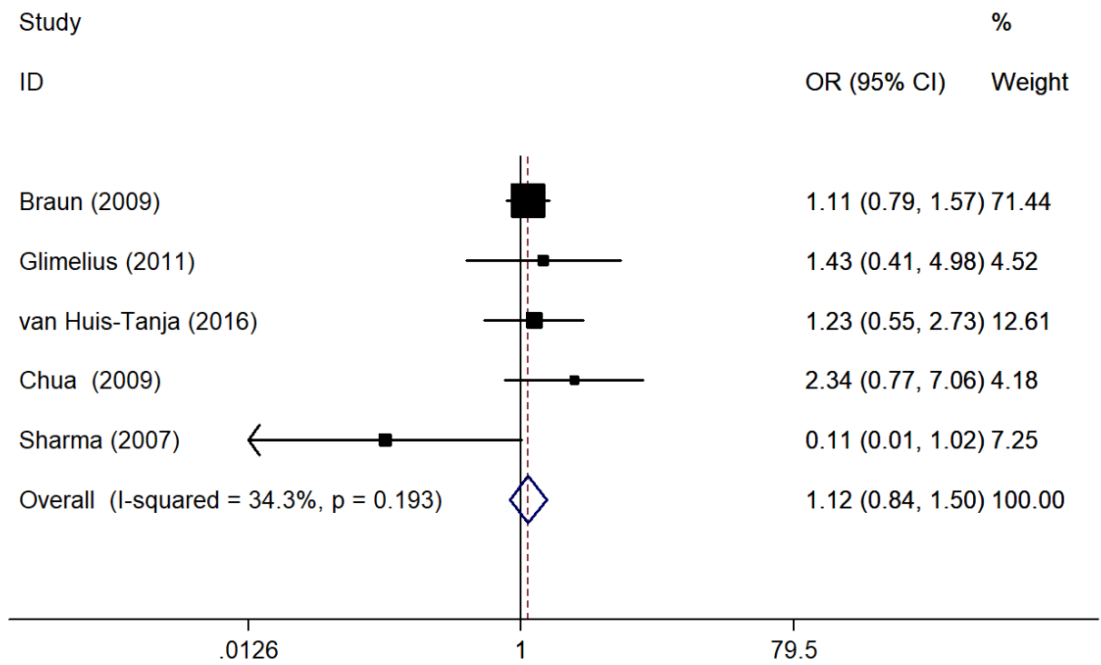

Figure 5: Forest plot of MTHFR gene rs1801133 C >T polymorphisms and grade 3-4 adverse reaction of colorectal cancer in recessive mutation model [TT vs $(\mathrm{CT}+\mathrm{CC})]$.

Table 2: The publication bias evaluation by Egger's line regression test.

\begin{tabular}{lllll}
\hline Model & Coef & $\mathbf{t}$ & $\mathbf{p}$ & $\mathbf{9 5 \% \mathrm { Cl }}$ \\
\hline TR_dominant & -1.64 & -0.54 & 0.60 & $-8.49 \sim 5.20$ \\
TR_recessive & 3.86 & 1.52 & 0.17 & $-2.15 \sim 9.87$ \\
AE_dominant & -0.25 & -0.41 & 0.70 & $-1.82 \sim 1.32$ \\
TR_recessive & 0.51 & 0.50 & 0.65 & $-2.75 \sim 3.78$ \\
\hline
\end{tabular}

\section{Grade 3-4 adverse reaction in recessive mutation model [TT vs $(\mathrm{CT}+\mathrm{CC})]$}

Without statistical heterogeneity, the data was pooled under a fixed effect model. The combined results indicated no statistical grade 3-4 adverse reaction between TT and $\mathrm{CT}+\mathrm{CC}$ groups (OR=1.12, 95\% CI: 0.84 1.50, $\mathrm{p}>0.05$ ), Figure 5.

\section{Publication bias analysis}

The publication bias of the included 16 studies was assessed by Begg's funnel plot (Figure 6) and Egger's line regression test. The funnel plots were general symmetrical which indicating no obvious publication bias. And the Eegger's line regression test also demonstrated no significant publication bias $\left(\mathrm{p}_{\text {all }}>0.05\right)$, Table 2 .

\section{Discussion}

Colorectal cancer (CRC) is one of the most diagnosed malignant tumor of the digestive system [23]. It was the fourth most diagnosed cancer in the United States with an estimated 143,460 new cases in the year 2012 [24]. The general prognosis of the early stage CRC was good in patients who received surgery. However, the 5-year survival rate was low even under the treatment of chemoradiotherapy for advanced stages of the disease. The most used chemotherapy regimen was fluorouracil (5-FU) based chemotherapy regimen for colorectal cancer patients $[4,25]$.

Folic acid is involved in the synthesis and transformation of nucleotides. Methylene tetrahydrofolate reductase (MTHFR), a key enzyme in folate metabolism, catalyzes the irreversible conversion of 5,10-methylene tetrahydrofolate (5,10-MTHF) to 5-methyltetrahydrofolate, which converts homocysteine to methionine and then to S-adenosylmethionine (SAM). The most common SNP site of MTHFR gene is rs $1801133 \mathrm{C}>\mathrm{T}$, where cytosine $\mathrm{C}$ mutates to thymine $\mathrm{T}(677 \mathrm{C}>\mathrm{T})$, resulting in alanine being replaced by valine, which reduces the activity of MTHFR [26-29]. 5-FU is transformed into fluorouracil deoxynucleotide (FdUMP) in cells with thymidylate synthase (TS) and 5,10MTHF, inhibiting the function of thymidylate synthase, preventing the conversion of deoxyuridine (dUMP) to deoxythymidine (dTMP), affecting DNA synthesis[30, 31]. Therefore, MTHFR gene rs1801133 C $>$ T polymorphisms can affect 5-FU base chemotherapy in patients with colorectal cancer. 

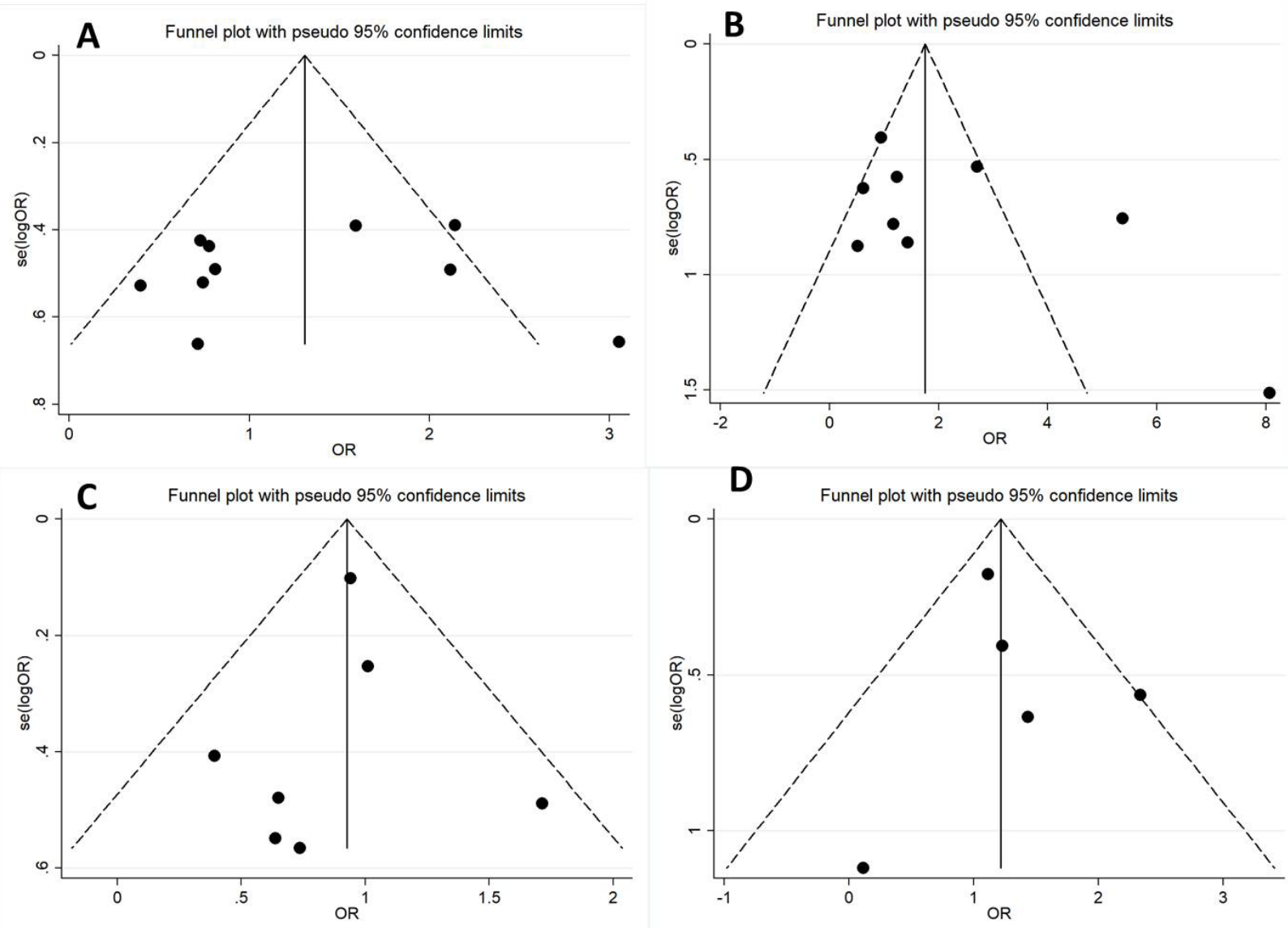

Figure 6: The funnel plot for publication bias evaluation.

Recently, several studies $[16,18,20]$ have discussed the correlation between MTHFR gene rs1801133 $\mathrm{C}>\mathrm{T}$ polymorphisms and response to $5-\mathrm{FU}$ based chemotherapy in patients with CRC. However, because of small sample size and weak statistical power, clinical evidence was weak. Therefore, we performed this metaanalysis in order to further evaluate the relationship between MTHFR rs1801133 C >T polymorphism and 5-FU based chemotherapy response in order to provide a more reliable evidence for the rational use of $5-\mathrm{FU}$ chemotherapy regimens in clinic. We found the response and adverse reaction of 5-FU based chemotherapy in colorectal patients was not different in terms of MTHFR rs1801133 C $>$ T polymorphisms.

Although we did not find any correlation between MTHFR rs1801133 C>T polymorphism and 5-FU based chemotherapy response or adverse reactions, the results demonstrated a trend that patients with TT/CT genotype were more prone to response to 5-FU based chemotherapy response and had less grade 3-4 adverse reactions.

\section{Conclusion}

Base on the present evidence, the response and adverse reactions of 5-FU based chemotherapy in colorectal patients were not different in terms of MTHFR rs1801133 $\mathrm{C}>\mathrm{T}$ polymorphisms. However, only 16 publications were included with a relative small sample size. More studies are needed to further discuss this correlation.

Conflict of interest: Authors state no conflict of interest

\section{Reference}

[1] Fang L, Guangyue Q, Tingyu T, et al. Methylenetetrahydrofolate Reductase (MTHFR) Gene rs1801133 C>T Polymorphisms and Lung Cancer Susceptibility: An Updated Meta-analysis[]]. Pteridines, 2019;30:65-73.

[2] Guo X, Fang W, Li Z, et al. Adjuvant radiotherapy, chemotherapy or surgery alone for high-risk histological node negative esophageal squamous cell carcinoma: Protocol for a 
multicenter prospective randomized controlled trial[J]. Thorac Cancer, 2018;9(12):1801-1806.

[3] Bleiberg H. Colorectal cancer--is there an alternative to 5-FU?[J]. Eur J Cancer, 1997;33(4):536-541.

[4] Lokich J. Infusional 5-FU for advanced colorectal cancer[]]. J Infus Chemother, 1995;5(4):208-211.

[5] Higgins JP, Thompson SG, Deeks JJ, et al. Measuring inconsistency in meta-analyses[J]. BMJ, 2003;327(7414):557560.

[6] Egger M, Davey SG, Schneider M, et al. Bias in metaanalysis detected by a simple, graphical test[J]. BM], 1997;315(7109):629-634.

[7] Cohen V, Panet-Raymond V, Sabbaghian N, et al. Methylenetetrahydrofolate reductase polymorphism in advanced colorectal cancer: a novel genomic predictor of clinical response to fluoropyrimidine-based chemotherapy[J]. Clin Cancer Res, 2003;9(5):1611-1615.

[8] Etienne MC, Formento JL, Chazal M, et al. Methylenetetrahydrofolate reductase gene polymorphisms and response to fluorouracil-based treatment in advanced colorectal cancer patients[]]. Pharmacogenetics, 2004;14(12):785-792.

[9] Jakobsen A, Nielsen JN, Gyldenkerne N, et al. Thymidylate synthase and methylenetetrahydrofolate reductase gene polymorphism in normal tissue as predictors of fluorouracil sensitivity[J]. J Clin Oncol, 2005;23(7):1365-1369.

[10] Marcuello E, Altés A, Menoyo A, et al. Methylenetetrahydrofolate reductase gene polymorphisms: genomic predictors of clinical response to fluoropyrimidinebased chemotherapy?[J]. Cancer Chemother Pharmacol, 2006;57(6):835-840

[11] Capitain O, Boisdron-Celle M, Poirier AL, et al. The influence of fluorouracil outcome parameters on tolerance and efficacy in patients with advanced colorectal cancer[]]. Pharmacogenomics J, 2008;8(4):256-267.

[12] Ruzzo A, Graziano F, Loupakis F, et al. Pharmacogenetic profiling in patients with advanced colorectal cancer treated with first-line FOLFIRI chemotherapy[J]. Pharmacogenomics ], 2008;8(4):278-288.

[13] Suh KW, Kim JH, Kim DY, et al. Which gene is a dominant predictor of response during FOLFOX chemotherapy for the treatment of metastatic colorectal cancer, the MTHFR or XRCC1 gene?[J]. Ann Surg Oncol, 2006;13(11):1379-1385.

[14] Sharma R, Hoskins JM, Rivory LP, et al. Thymidylate synthase and methylenetetrahydrofolate reductase gene polymorphisms and toxicity to capecitabine in advanced colorectal cancer patients[]]. Clin Cancer Res, 2008;14(3):817-825.

[15] Braun MS, Richman SD, Thompson L, et al. Association of molecular markers with toxicity outcomes in a randomized trial of chemotherapy for advanced colorectal cancer: the FOCUS trial[J]. J Clin Oncol, 2009;27(33):5519-5528.

[16] Chua W, Goldstein D, Lee CK, et al. Molecular markers of response and toxicity to FOLFOX chemotherapy in metastatic colorectal cancer[J]. Br J Cancer, 2009;101(6):998-1004. DOI: $10.1038 /$ sj.bjc.6605239.

[17] Etienne-Grimaldi MC, Milano G, Maindrault-Goebel F, et al. Methylenetetrahydrofolate reductase (MTHFR) gene polymorphisms and FOLFOX response in colorectal cancer patients[J]. Br J Clin Pharmacol, 2010;69(1):58-66.
[18] Glimelius B, Garmo H, Berglund A, et al. Prediction of irinotecan and 5 -fluorouracil toxicity and response in patients with advanced colorectal cancer[J]. Pharmacogenomics J, 2011;11(1):61-71. DOI: 10.1038/tpj.2010.10.

[19] van Huis-Tanja LH, Gelderblom H, Punt CJ, et al. MTHFR polymorphisms and capecitabine-induced toxicity in patients with metastatic colorectal cancer[]]. Pharmacogenet Genomics, 2013;23(4):208-218.

[20] Kumamoto K, Ishibashi K, Okada N, et al. Polymorphisms of GSTP1, ERCC2 and TS-3'UTR are associated with the clinical outcome of mFOLFOX6 in colorectal cancer patients[J]. Oncol Lett, 2013;6(3):648-654.

[21] Dong-ya S, Hai-tang X, Yao C, et al. Association of MTHFR, GSTP1, ERCC1 polymorphisms and response to FOLFOX in colorectal cancer patients[]]. Chinese Journal of Clinical Pharmacology and Therapeutics, 2015;20(01):75-81+85.

[22] Qiu-mei D, Ying LI, Sai-hua H. Prognostic Impact of MTHFR Polymorphism on Colorectal Cancer Patients Ttreated by Chemotherapy[J]. Journal of Sun Yat-sen University(Medical Sciences), 2016;37(1):95-99.

[23] Siegel RL, Miller KD, Fedewa SA, et al. Colorectal cancer statistics, 2017[J]. CA Cancer J Clin, 2017;67(3):177-193.

[24] Torre LA, Bray F, Siegel RL, et al. Global cancer statistics, 2012[J]. CA Cancer J Clin, 2015;65(2):87-108.

[25] Tsuji T. [Response marker to 5-FU after curative surgery in colorectal cancer][J]. Nihon Rinsho, 2003;61 Suppl 7:325-328.

[26] Luo Z, Lu Z, Muhammad I, et al. Associations of the MTHFR rs1801133 polymorphism with coronary artery disease and lipid levels: a systematic review and updated meta-analysis[J]. Lipids Health Dis, 2018;17(1):191.

[27] Zhang Y, Jia H, Wang S, et al. Cumulative review and metaanalyses on the association between MTHFR rs1801133 polymorphism and breast cancer risk: a pooled analysis of 83 studies with 74,019 participants[]]. Minerva Med, 2017;108(1):57-73.

[28] Li K, Yp H, Yang Z, et al. Association between MTHFR Ala222Val (rs1801133) polymorphism and bladder cancer susceptibility: a systematic review and meta-analysis[J]. Tumour Biol, 2013;34(5):2565-2572.

[29] Yu L, Chen J. Association of MTHFR Ala222Val (rs1801133) polymorphism and breast cancer susceptibility: An update meta-analysis based on 51 research studies[J]. Diagn Pathol, 2012;7:171.

[30] Sasaki T. [High-dose leucovorin and 5-FU][J]. Gan To Kagaku Ryoho, 1992;19(7):954-962.

[31] Mandel HG. The target cell determinants of the antitumor actions of 5-FU: does FU incorporation into RNA play a role?[J]. Cancer Treat Rep, 1981;65 Suppl 3:63-71. 